\title{
Reduction of Orbital Angular Momentum Radio Beam Divergence Using a 3D Printed Planar Graded Index Lenses
}

\author{
B Allen ${ }^{12}$, T.D Drysdale ${ }^{3}$, S Zhang ${ }^{4}$, D Isakov ${ }^{5}$, A Tennant ${ }^{6}$, W Whittow ${ }^{4}$, C. Stevens ${ }^{2}$, J Vardaxoglou ${ }^{4}$, J Coon $^{2}$ \\ ${ }^{1}$ Network Rail, The Quadrant:MK, Elder Gate, Milton Keynes, MK9 1ER, UK, Ben.Allen@networkrail.co.uk \\ ${ }^{2}$ Department of Engineering Science, University of Oxford, Parks Road, Oxford, OX1 3PJ, UK \\ ${ }^{3}$ The School of Engineering and Innovation, The Open University, Milton Keynes, MK7 6AA, UK \\ ${ }^{4}$ Wolfson School of Mechanical, Electrical and Manufacturing Engineering, Loughborough University, Loughborough, LE11 $3 T U$, UK \\ ${ }^{5}$ Department of Materials, University of Oxford, Parks Road, Oxford, OX1 3PH, UK \\ ${ }^{6}$ Department of Electronic and Electrical Engineering, University of Sheffield, Mappin Street, Sheffield, S1 3JD,UK
}

\begin{abstract}
This paper shows that the range of an Orbital Angular Momentum (OAM) radio link can be at least doubled by using a 3D graded index lens to reduce beam divergence. This is a major step towards realizing practical high capacity OAM radio links using cheap, compact and light weight lenses.
\end{abstract}

Index Terms - orbital angular momentum radio, 3D printed lens, antenna arrays.

\section{INTRODUCTION}

Recently, Orbital Angular Momentum (OAM) radio has attracted attention as a means of significantly increasing the capacity of line-of-sight radio links [1][2]. Specific applications include wireless connectivity for data centers, campus connectivity, and satellite networks and telecommunications backhaul such as along a road or rail corridor. This additional capacity is achieved by means of multiplexing parallel data streams through several independent OAM 'modes' operating on the same frequency. Generation of OAM modes can be by means of illuminating spiral phase plates [3] or using circular antenna arrays with appropriate phasing between elements [4]. The experimental OAM radio multiplexing system in [2] is shown to provide a data rate of $32 \mathrm{~Gb} / \mathrm{s}$ at a $28 \mathrm{GHz}$ carrier frequency, but required transmit and receive apertures of around $2 \mathrm{~m}$, and achieved a transmission range of $2 \mathrm{~m}$. OAM radio differs from conventional Multiple-Input Multiple-Output (MIMO) radio communications in that it suits line-of-sight links that, unlike MIMO, do not exhibit multipath diversity. OAM modes are independent from polarization in that they exploit spatial phase patterns produced by the emitter rather than the orientation of the electromagnetic fields. Traditional radio links use OAM mode zero. For OAM modes other than zero, a vortex exists in the center of the beam and rapid divergence of the beam occurs, which means that very large receive apertures are required for effective reception [5], making practical link ranges difficult to achieve. In [6], an OAM mode was generated using a split parabola antenna where the collimation due to the parabola helped to overcome divergence. In this case the antenna configuration could only support a single mode and in this case was not used to send data. It also requires the realization of a metallic parabola and feed structure making the equipment bulky and costly [7].

This paper reports experimental results obtained using an 8-element circular array as described in [4] together with a $3 \mathrm{D}$ printed planar graded index lens described in [8] in order to reduce the beam divergence and hence extend the OAM link range. This is a major step towards realizing OAM radio links over useful ranges. The lens is inexpensive, conveniently realizable, compact and light weight. With a suitable feed structure to the array, the configuration may also support several modes. A series of measurements have been performed in an anechoic chamber in order to determine the effect of placing this lens in front of the circular array in terms of beam directivity and link distance.

The following section describes the array, lens and experiment configuration. Results are then shown for several array / lens separation distances and expressed in terms of radiation pattern, beam divergence, gain and directional behavior. This has allowed the effect of the lens on link distance to be determined and conclusions to be drawn.

\section{SYSTEM CONFIGURATION}

The OAM array, as described in [4], consists of 8 rectangular patch elements. The array radius is set to 0.6 wavelengths to minimize the inter-element mutual coupling. The circular OAM array has an overall diameter of $6 \mathrm{~cm}$ and is manufactured from $0.16 \mathrm{~cm}$ thick FR4 circuit board with a relative permittivity of 4.3 . The incremental phase shift between adjacent elements is $-45^{\circ}$ to produce an OAM mode number of $l=-1$. The phase shifts were implemented using a microstrip corporate feeding network. The fabricated array and phase weighting scheme is showed in Fig la.

The planar graded index dielectric lens, as described in [8], was 3D-printed by using a fused deposition modelling 3D-printer. The lens comprised 6 concentric cylindrical rings with different infill factors. The non-solid in-fills produced the bespoke graded effective permittivities. These tightly fitted concentric rings in the lens were 3D-printed in one single-process using thermoplastic polylactic acid (PLA). The permittivity and the loss tangent of the solid PLA was characterized as 2.7 and 0.008 at $10 \mathrm{GHz}$ [8]. The lens has a 
diameter of $12 \mathrm{~cm}$ and thickness of $1.85 \mathrm{~cm}$, as shown in Fig. 1b. It is designed for a focal length of $15 \mathrm{~cm}$. The behavior of the lens when illuminated by a horn antenna is described in [8], and shown to produce an additional gain of up to $11 \mathrm{dBi}$ at the focal length of $15 \mathrm{~cm}$. In this case, it is illuminated by the OAM circular array. Due to the vortex at the center of the array's OAM mode $l=-1$ beam, the center of the lens receives minimum radiation when compared to the horn, where signals radiated from the horn may be considered as OAM mode zero.

An azimuth-plane scan was set up for measuring the radiation patterns of various configurations of the OAM array and the lens. This system scanned the received power from $-90^{\circ}$ to $+90^{\circ}$ in the azimuth-plane to generate the front half of the radiation pattern. An X-band pyramidal horn antenna was mounted on a track and revolved around the lens with $1.5 \mathrm{~m}$ radius to measure the received power. This procedure was repeated for several array / lens separation distances of $3 \mathrm{~cm} \leq d \leq 15 \mathrm{~cm}$. This procedure was then repeated with $\mathrm{d}$ selected to be $8 \mathrm{~cm}$, i.e., where the null width is at a minimum, and with the distance between the lens and horn varied from $0.66 \mathrm{~m}$ to $3.52 \mathrm{~m}$. The data was used to determine the beam divergence with and without the lens.

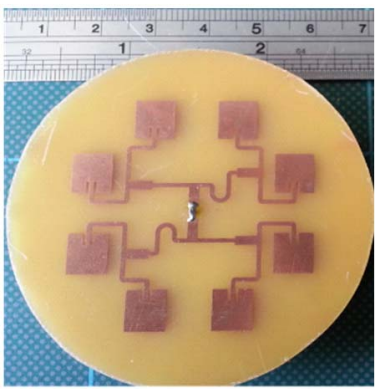

$a$.

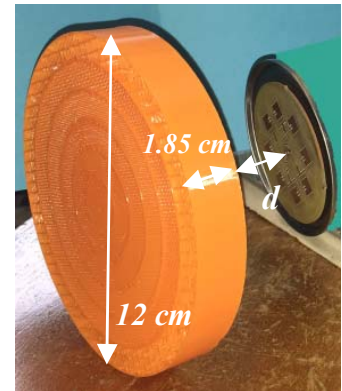

$b$.
Fig. 1 OAM circular phased array and planar lens.

a. Realized OAM array antenna on FR4 (mode -1) with elemental phase increments shown. $b$. 3D printed planar graded index lens and array combination with dimensions shown.

\section{RESULTS}

Fig. 2 shows the directivity for the array without lens and for array - lens separation distances $(d)$ of 15, 8, 4 and $2 \mathrm{~cm}$. It is clear from the figure that the introduction of this lens improves the directivity for values of $\mathrm{d}$ except for when $d=$ $8 \mathrm{~cm}$. For the case of $d=15 \mathrm{~cm}$, it is seen that, although the lens is designed for a focal distance of $15 \mathrm{~cm}$, the directivity is not maximized. This is because the array does not illuminate the lens with a single source at the center. Instead, there are eight sources, i.e., one from each element, that are in a circle of radius $1.5 \mathrm{~cm}$ around the central axis as depicted in Fig. 1a hence each source illuminated the lens from an off-set position. It can also be observed that the null width varies with $\mathrm{d}$, as does the null location which is attributed to small variations in the alignment of the array and lens occurring between measurements. The measurements data has been used to determine the maximum directivity, angle of maximum directivity and the null width (defined as the angle between maxima) for each value of $d$. These are tabulated in Table 1. The table shows the minimum null width occurring at $d=8 \mathrm{~cm}$ and maximum directivity when $d=4 \mathrm{~cm}$.

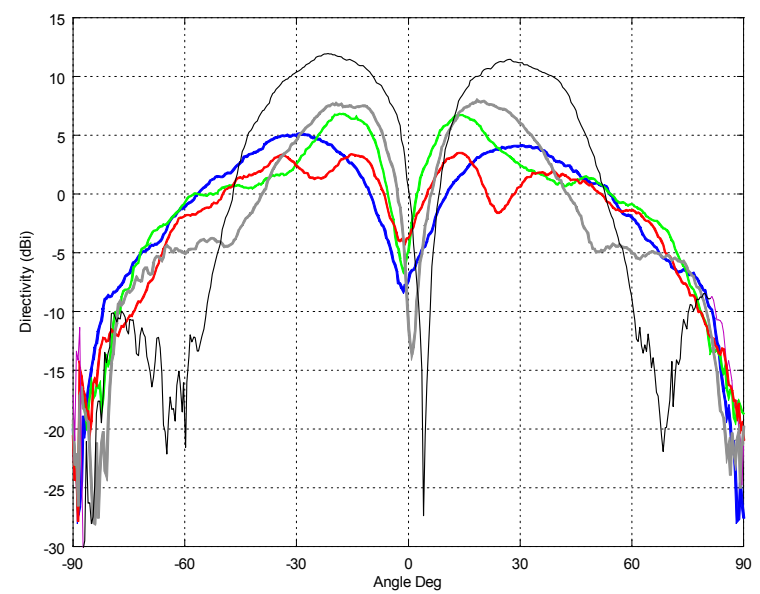

Fig 2. Measured directivity of OAM array and lens configuration: BLUE Array only, RED $15 \mathrm{~cm}$, GREEN $8 \mathrm{~cm}$, Grey $4 \mathrm{~cm}$, Black $2 \mathrm{~cm}$.

Table 1. Maximum directivity and null width for various values of $d$

\begin{tabular}{|l|c|c|c|c|c|}
\hline \multirow{2}{*}{} & \multicolumn{5}{|c|}{$d(\mathbf{c m})$} \\
\cline { 2 - 6 } & $\begin{array}{c}\text { Array } \\
\text { only }\end{array}$ & $\mathbf{1 5}$ & $\mathbf{8}$ & $\mathbf{4}$ & $\mathbf{2}$ \\
\hline Max directiviy (dBi) & 5.1 & 7.0 & 3.5 & 12.0 & 9.0 \\
\hline Ang. of Max Dir (deg) & -29.3 & -17.6 & 14.0 & 18.5 & -21.6 \\
\hline Null width (deg) & 59.9 & 31.5 & 29.3 & 37.8 & 48.6 \\
\hline
\end{tabular}

Fig. 3 shows the phase and amplitude profiles of the $\mathrm{x}-\mathrm{y}$ plane of the OAM array with and without the lens. The lens / array seperation of $d=4 \mathrm{~cm}$, i.e., maximum directivity has been used, and the OAM array / measurement antenna seperation was $45 \mathrm{~cm}$. The measurement antenna was stepped across a $220 \times 218 \mathrm{~mm}$ grid in $2 \mathrm{~mm}$ steps, where the amplitude and phase was recorded using a vector network analyser for each location. The figure shows the improved directivity achieved with the lens in place, and that the phase profile is preserved. 

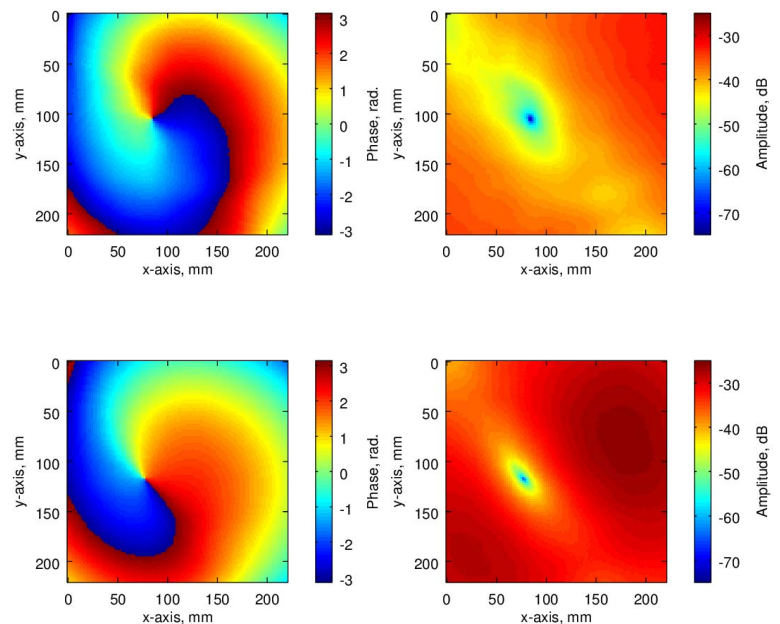

Fig

3. Phase and amplitude profiles of the $x-y$ plane of the OAM array without (top) and with (bottom) the lens.

Fig. 4 plots the profile of the signal maxima with and without the lens as a function of distance from the transmitter (Tx) ranging from $0.66 \mathrm{~m}$ to $3.52 \mathrm{~m}$. A best-fit line between the points is also shown and determined using a 1st order polynomial fit with least mean squared error. Thus, a beam divergence of $56^{\circ}$ is shown without the lens, and $24^{\circ}$ with the lens. Consequently, when a lens is used, an aperture of $1.5 \mathrm{~m}$ is required to receive the signal maxima at a distance of $3.5 \mathrm{~m}$. This aperture size would only be sufficient at $1.5 \mathrm{~m}$ without a lens. Thus the link distance is approximately doubled for a given aperture when this lens is used at one end of a link, and quadrupled when at both ends. Further improvements are expected by optimising the lens design by taking into account the nature of the illumination of the lens by the OAM array. The above results indicate the positive impact of the lens on achievable link distance, marking a major step towards realising useful link distances when OAM modes are employed.

\section{CONCLUSIONS}

This paper has demonstrated the feasibility of using 3D printed planar graded index lens for collimating diverging OAM radio beams, and hence at least doubling the achievable link distance. This opens up the possibility for the realization of practical OAM radio links using cheap, compact and light weight lenses.

\section{ACKNOWLEDGMENTS}

Ben Allen is grateful for the support of the Royal Society Industrial Fellowship scheme under award number IF160001. The authors acknowledge the support of EPSRC grant EP/N010493 SYMETA project that enabled the development of the lens.

\section{REFERENCES}

[1] S.M Mohammadi, et al., "Orbital angular momentum in radio-a system study”, IEEE Trans. Antennas Propag. 58, 565-572 (2010), doi: 10.1109/TAP.2009.2037701

[2] Y Yan, G Xie et al, "High-capacity millimetre-wave communications with orbital angular momentum multiplexing", Nature Communications, Vol. 5, Article number: 4876, 2014, doi:10.1038/ncomms5876

[3] F.E Mahmouli, S Walker "4-Gbps Uncompressed Video Transmission over a 60-GHz Orbital Angular Momentum Wireless Channel", IEEE Wireless Coms. Letters, Vol. 2, Iss. 2, 2013, pp223 226, doi: 10.1109/WCL.2013.012513.120686

[4] Q Bai, A. Tennant, B. Allen, "Experimental circular phased array for generating OAM radio beams", IET Electronics Letters, 2014, Vol. 50, Issue 20, Pages: 1414 - 1415, DOI: 10.1049/el.2014.2860

[5] M. J Padgett, F. M Miatto, M. P. J. Lavery, A. Zeilinger, R. W. Boyd, "Divergence of an orbital-angular-momentum-carryingbeam upon propagation,” New J. Phys., vol. 17, no. 2, p. 023011, Feb. 2015, New J. Phys, doi:10.1088/1367-2630/17/2/023011.

[6] F Tamburini et al, "Encoding many channels on the same frequency through radio vorticity: first experimental test", New Journal on Physics, Vol. 14, 2012, doi: 10.1088/1367-2630/14/3/033001

[7] W Zhang, S Zheng, X Hui, R Dong, X Jin, H Chi, X Zhang, "Mode Division Multiplexing Communication Using Microwave Orbital Angular Momentum: An Experimental Study”, IEEE Trans. Wireless Communications. Vol. 16, No. 2, Feb. 2017, pp1308-1318, doi: 10.1109/TWC.2016.2645199

[8] S Zhang, R.K Arya, S Pandey, Y Vardaxoglou, W Whittow, R Mittra, "3D-printed planar graded index lenses", IET Microwaves, Antennas \& Propagation, 2016, Vol. 10, Iss. 13, Pages: 1411 - 1419, DOI: 10.1049/iet-map.2016.0013, doi: 10.1049/iet-map.2016.0013

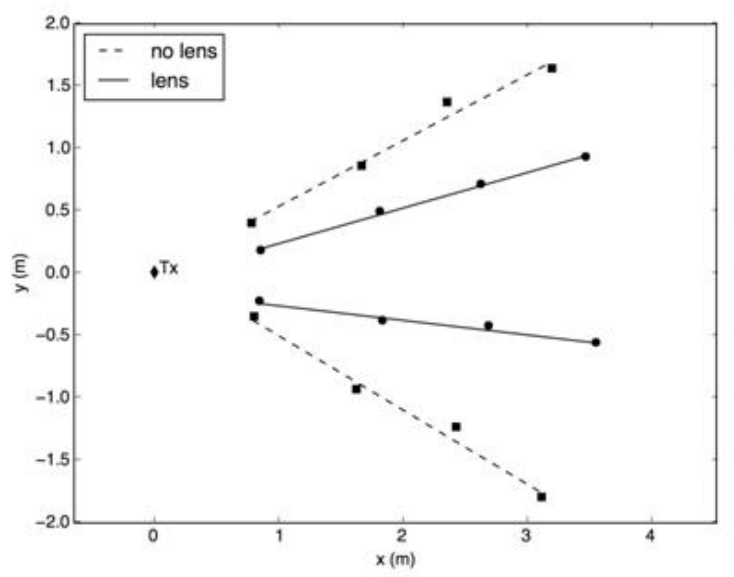

Fig. 4. Lines of signal maxima with and without a lens based on measured results. Curved arrows indicate divergence angles, straight arrows indicate aperture diameters. 